\title{
Prosultiamine treatment as a new therapeutic strategy in human $T$ lymphotropic virus type l-associated myelopathy/tropical spastic paraparesis
}

Human T lymphotropic virus type I (HTLV-I)-associated myelopathy/tropical spastic paraparesis (HAM/ TSP) is a chronic myelopathy characterized by motor dysfunction of the lower extremities and urinary disturbance. ${ }^{1}$ The primary neuropathological feature of HAM/TSP is chronic inflammation in the spinal cord caused by high HTLV-I proviral load in peripheral blood mononuclear cells (PBMC). Therefore, immunomodulatory therapy, such as corticosteroid hormones and interferon- $\alpha$, has been the main treatment for HAM/TSP patients. ${ }^{2}$ However, there are many issues in long-term treatment with these drugs, such as insufficient effects and various sideeffects. Once the myelopathy develops, the main neurological symptoms, such as motor dysfunction of the lower extremities accompanied by urinary disturbance, are progressive and lead to a deterioration in the quality of life of patients. Therefore, novel and safe therapeutic regimens are urgently required for HAM/TSP patients to use as a treatment, or prevent disease progression.

Prosultiamine (Alinamin), a vitamin $\mathrm{B}_{1}$ derivative, is safely available in Japan for the treatment of Wernicke's encephalopathy and polyneuropathy induced by deficiency of vitamin $\mathrm{B}_{1}$. Based on the data that prosultiamine can induce the caspase-dependent apoptosis of HTLV-I-infected cells through disruption of intracellular redox reactions by a disulfide moiety in its structure, ${ }^{3}$ we carried out a clinical trial with prosultiamine for $24 \mathrm{HAM}$ /TSP patients using an open-labeled design. Here, I will show the remarkable efficacy of prosultiamine treatment against HAM/TSP patients without serious adverse effects. ${ }^{4,5}$

Prosultiamine $300 \mathrm{mg}$ was given orally once daily for 12 weeks. As a result, improvement in the motor function of the lower extremities based on a reduction in spasticity (e.g. decrease in time required for walking and descending a flight of stairs) was observed. Interestingly, this treatment induced the striking amelioration of urinary disturbance. In an urodynamic study (UDS), bladder capacity and detrusor pressure, and then maximum flow rate, increased significantly. Detrusor overactivity and detrusor-sphincter dyssynergia improved in 68.8\% and $45.5 \%$ of patients, respectively. Improvement in UDS corresponded with improvements in the score of nocturia quality of life questionnaire. Thus, given that the mean duration of illness of the patients enrolled in the present study was relatively long (approximately 21 years), the efficacy of this treatment is promising.

In the present study, HTLV-I proviral copy numbers in $\mathrm{PBMC}$ decreased significantly (approximately $15.4 \%$ ) compared with pretreatment levels. However the remarkable clinical improvement in the present study cannot be attributed solely to a decrease in HTLV-I proviral copy numbers in PBMC. Although the exact mechanism is not known, it might be conceivable that, as one of the mechanisms, prosultiamine functions to induce the apoptosis of HTLV-Iinfected cells by the disruption of intracellular redox system in the spinal cord, even if the extent of reduction of the number of HTLV-I-infected cells in PBMC is relatively small. Further investigations including analysis of cerebrospinal fluid are required to elucidate the exact mechanism of action of prosultiamine.

Overall, the present results suggest that prosultiamine could be a new promising therapeutic tool for HAM/TSP patients. Therefore, further studies are warranted for the evaluation of prosultiamine treatment against HAM/TSP in a large-scale, randomized, controlled study and long-term treatment.

\section{Competing interests}

None.

\section{References}

1. Osame M, Matsumoto M, Usuku K, Izumo S, Ijichi N, Amitani $\mathrm{H}$, et al. Chronic progressive myelopathy associated with elevated antibodies to human T-lymphotropic virus type I and adult T-cell leukemia-like cells. Ann Neurol. 1987; 21: 117-22.

2. Nakamura T, Nishiura Y, Eguchi K. Therapeutic strategies in HTLV-I-associated myelopathy/tropical spastic paraparesis 
(HAM/TSP). Cent Nerv Syst Agents Med Chem. 2009; 9: 137-49.

3. Nishiura $Y$, Nakamura T, Fukushima $N$, Nakamura $H$, Ida $\mathrm{H}$, Aramaki $\mathrm{T}$, et al. Disulfide-mediated apoptosis of human T-lymphotropic virus type-I (HTLV-I)-infected cells in patients with HTLV-I-associated myelopathy/tropical spastic paraparesis. Antivir Ther. 2009; 14: 533-42.

4. Nakamura T, Matsuo T, Fukuda T, Yamato S, Yamaguchi K, Kinoshita I, et al. Efficacy of prosultiamine treatment in patients with human T lymphotropic virus type I-associated myelopathy/tropical spastic paraparesis: results from an open-label clinical trial. BMC Med. 2013; 11: 182.

5. Kira J. Therapeutic benefits of an oral vitamin B1 derivative for HAM/TSP. BMC Med. 2013; 11: 183.

Tatsufumi Nakamura Department of Molecular Microbiology and Immunology, Graduate School of Biomedical Sciences, Nagasaki University, Nagasaki, Japan 